1 Hacettepe Journal of Mathematics and Statistics

h Volume 45 (2) (2016), 483-495

\title{
Numerical computation and properties of the two dimensional exponential integrals
}

\author{
Şeyhmus Yardımcı ${ }^{* \dagger}$, Murat Olgun ${ }^{\ddagger}$ and Çağla Can ${ }^{\S}$
}

\begin{abstract}
In this paper, we investigate problem of convergence of the twodimensional exponential integral (TDEI) functions arising in the study of the radiative transfer in a multi-dimensional medium. In our study, generalized exponential integral function's ( GEIF ) are expressed with double improper integrals as given in the original expression. Then we study the properties and asymptotic behaviour of the TDEI functions. We also give numerical computations of the values of TDEI functions.
\end{abstract}

Keywords: Numerical computations, exponential integrals, uniform convergence, multi-dimensional radiative transfer.

2000 AMS Classification: 33F05, 65Z05, 40A10

Received: 26.11.2014 Accepted : 27.02.2015 Doi : 10.15672/HJMS.20164512505

\section{Introduction}

The two-dimensional exponential integral (TDEI) functions play an important role in various fields of theoretical physics, quantum chemistry, theory of transport process, theory of fluid flow and theory of radiative transfer in a multi-dimensional medium [6], [7], [11]-[13], [19], [22]. The TDEI functions are especially useful for the study of anisotropic scattering in a two-dimensional medium with a scattering phase function [12], [13], [22]. Breig and Crosbie derived a series expansion and recurrence relations suitable for numerical computation of the one-dimensional exponential integral functions [7]. It is shown that the absorption of solar radiation by the earth's atmosphere is given in terms of first-order exponential integral function. The fundamental integral equation of the radiative transfer of two-dimensional planar media with anisotropic scattering was derived

\footnotetext{
*Department of Mathematics, Ankara University, 06100 Tandoğan, Ankara, TURKEY, Email : smyardimci@ankara.edu.tr

${ }^{\dagger}$ Corresponding Author.

${ }^{\ddagger}$ Department of Mathematics, Ankara University, 06100 Tandoğan, Ankara, TURKEY, Email : olgun@ankara.edu.tr

${ }^{\S}$ Department of Mathematics, Ankara University, 06100 Tandoğan, Ankara, TURKEY, Email : cglcan@hotmail.com
} 
by Crosbie and Dougherty [11]. Note that the TDEI functions are the kernel of that integral equation. The TDEI functions play an important role in the investigation of the two-dimensional radiative transfer in an absorbing-emitting cylindrical medium and determination of the radiative flux [12]. The generalized exponential integral functions are studied in [1]-[5], [10], [17]. In [2] GEIF' 's are expressed with the single integrals. In our study, GEIF 's are expressed with double improper integrals as given in the original expression. This depends on the truth that the uniform convergence of integrals gives more precise results. Also in [2] GEIF 's are given In terms of Bessel functions, in the form of series. This GEIF' 's approximation gives ruder results compared to ours. This study uses a different methodology from [1], [2], [10], [17] and results are achieved with higher accuracy. The TDEI functions examined in this work are defined as

$$
\varepsilon_{n}(\tau, \beta)=\frac{\tau^{n-1}}{2 \pi} \int_{-\infty}^{\infty} \int_{-\infty}^{\infty}\left[\exp (-r) / r^{n+1}\right] \exp (-i \beta x) d x d y
$$

where $r^{2}=x^{2}+y^{2}+\tau^{2}$ and $n=1,2, \ldots$.

Note that the TDEI functions are two-dimensional analogs of the exponential integral functions [14].

$$
E_{n}(\tau)=\int_{1}^{\infty} t^{-n} \exp (-\tau t) d t
$$

$n=1,2, \ldots$. The exponential integral function (1.2) plays an important role in various fields of theoretical physics, quantum chemistry and theory of transport process [8], [9], [16], [20], [21].

Many properties of the TDEI functions depend on the uniform convergence of the improper integral (1.1). In this paper, we study the problem of convergence of the TDEI functions $\varepsilon_{n}(\tau, \beta)$. We also investigate the properties, asymptotic behaviour and numerical computation of the TDEI functions.

\section{Uniform convergence}

Let us consider the improper integral

$$
\int_{-\infty}^{\infty} \int_{-\infty}^{\infty} f(\tau, \beta, x, y) d x d y
$$

where $(\tau, \beta) \in D \subset \mathbb{R}^{2}$.

2.1. Definition. [18]. Integral (2.1) is said to be uniformly convergent with respect to $(\tau, \beta) \in D$ if it is convergent for all $(\tau, \beta)$ and if, given any $\varepsilon>0$, there is a sufficiently large number $R_{0}$ independent of $(\tau, \beta)$ and such that for any $R$ satisfying the inequality $R>R_{0}$ there holds the inequality

$$
\left|\int_{R^{2}-\omega_{R}} \int_{f} f(\tau, \beta, x, y) d x d y\right|<\varepsilon
$$

where $\omega_{R}$ is the ball of radius $R$ with centre of the origin.

2.2. Theorem. [18]. If for the function $f(\tau, \beta, x, y)$ in question there holds the inequality

$$
|f(\tau, \beta, x, y)| \leq \varphi(x, y),(\tau, \beta) \in D
$$


and the improper integral

$$
\int_{-\infty}^{\infty} \int_{-\infty}^{\infty} \varphi(x, y) d x d y
$$

is convergent then integral (2.1) convergences uniformly with respect to $(\tau, \beta)$ on $D$.

For all $\varepsilon>0$ we define the domain

$$
D(\varepsilon)=\{(\tau, \beta) \in \Omega, \tau \in[\varepsilon, \infty), \beta \in(-\infty, \infty)\},
$$

where

$$
\Omega=\left\{(\tau, \beta) \in \mathbb{R}^{2}, \tau \in[0, \infty), \beta \in(-\infty, \infty)\right\}
$$

2.3. Theorem. i) The two-dimensional exponential integral function $\varepsilon_{n}(\tau, \beta)$ is uniformly convergent with respect to $(\tau, \beta)$ on $D(\varepsilon)$.

ii) The function $\varepsilon_{n}(\tau, \beta)$ is nonuniformly convergent with respect to $(\tau, \beta)$ on $\Omega$.

Proof. i)

$$
\varepsilon_{n}(\tau, \beta)=\frac{\tau^{n-1}}{2 \pi} \int_{-\infty}^{\infty} \int_{-\infty}^{\infty}\left[\exp (-r) / r^{n+1}\right] \exp (-i \beta x) d x d y
$$

where $r^{2}=x^{2}+y^{2}+\tau^{2}$.

If we define

$$
g(\tau, \beta, x, y)=\left[\exp \left(-\sqrt{x^{2}+y^{2}+\tau^{2}}\right) /\left(\sqrt{x^{2}+y^{2}+\tau^{2}}\right)^{n+1}\right] \exp (-i \beta x)
$$

then

$$
\varepsilon_{n}(\tau, \beta)=\frac{\tau^{n-1}}{2 \pi} \int_{-\infty}^{\infty} \int_{-\infty}^{\infty} g(\tau, \beta, x, y) d x d y
$$

For all $\varepsilon>0$ we have

$$
|g(\tau, \beta, x, y)| \leq \exp \left(-\sqrt{x^{2}+y^{2}+\varepsilon^{2}}\right) / \varepsilon^{n} \sqrt{x^{2}+y^{2}+\varepsilon^{2}}
$$

and

$$
\begin{aligned}
& \int_{-\infty}^{\infty} \int_{-\infty}^{\infty} \exp \left(-\sqrt{x^{2}+y^{2}+\varepsilon^{2}}\right) / \varepsilon^{n} \sqrt{x^{2}+y^{2}+\varepsilon^{2}} d x d y \\
& =2 \pi \varepsilon^{-n} \exp (-\varepsilon)<\infty .
\end{aligned}
$$

So from the Theorem 2.1 we find that the function $\varepsilon_{n}(\tau, \beta)$ is uniformly convergent with respect to $(\tau, \beta)$ on $D(\varepsilon)$.

ii) For all $(\tau, \beta) \in \Omega$ we have

$$
\begin{aligned}
\varepsilon_{n}(\tau, \beta) & =\frac{\tau^{n-1}}{2 \pi} \int_{-\infty}^{\infty} \int_{-\infty}^{\infty}\left[\exp (-r) / r^{n+1}\right] \exp (-i \beta x) d x d y \\
& >-\frac{\tau^{n-1}}{2 \pi} \int_{-\infty}^{\infty} \int_{-\infty}^{\infty} \exp (-r) / r^{n+1} d x d y
\end{aligned}
$$


Let us consider

$$
\int_{-\infty}^{\infty} \int_{-\infty}^{\infty} \varphi(\tau, x, y) / r^{n+1} d x d y
$$

where $\varphi(\tau, x, y)=\exp (-r)$.

$\varphi(\tau, 0,0)=\exp (-\tau)>0$. Then there is a ball $\omega$ with centre at the origin and radius so small that $|\varphi(\tau, x, y)|>\frac{|\varphi(\tau, 0,0)|}{2}=\frac{\exp (-\tau)}{2}>0$ on that ball.

Therefore

$$
\begin{aligned}
\left|\iint_{\omega} \varphi(\tau, x, y) / r^{n+1} d x d y\right| & =\iint_{\omega}|\varphi(\tau, x, y)| / r^{n+1} d x d y \\
& >\frac{\exp (-\tau)}{2} \iint_{\omega} 1 / r^{n+1} d x d y \rightarrow \infty \text { for } \tau \rightarrow 0
\end{aligned}
$$

Therefore the function $\varepsilon_{n}(\tau, \beta)$ is nonuniformly convergent with respect to $(\tau, \beta)$ on $\Omega$.

\section{Properties of the TDEI function}

Let $G \in \mathbb{R}_{m}$ and $D \in \mathbb{R}_{n}$ where $\mathbb{R}_{m}$ and $\mathbb{R}_{n}$ denote $m$-dimensional and $n$-dimensional spaces, respectively.

We shall consider an integral of the form

$$
F(\xi)=\int_{D} f(\xi, \eta) d \eta \quad(\xi \in G)
$$

taken over an unbounded domain $D$ such that it has the point at infinity as its only singularity for any $\xi \in G$.

3.1. Theorem. [18]. If the function $f(\xi, \eta)$ is continuous on

$$
G \times D:=\{\xi \in G, \eta \in D\}
$$

and if the integral (3.1) is uniformly convergent with respect to $\xi$ on $G$, then the function

$$
F(\xi)=\int_{D} f(\xi, \eta) d \eta
$$

is a continuous function with respect to $\xi$ on $G$ and for all $\xi_{0} \in G$

$$
\lim _{\xi \rightarrow \xi_{0}} \int_{D} f(\xi, \eta) d \eta=\int_{D} \lim _{\xi \rightarrow \xi_{0}} f(\xi, \eta) d \eta .
$$

3.2. Theorem. [18]. If the conditions of Theorem 3.1 hold then the function $F(\xi)$ can be integrated with respect to $\xi$ on $G$ under the integral sign, that is

$$
\int_{G} F(\xi) d \xi=\int_{G} d \xi \int_{D} f(\xi, \eta) d \eta=\int_{D} d \eta \int_{G} f(\xi, \eta) d \xi
$$

3.3. Theorem. The function $\varepsilon_{n}(\tau, \beta)$ is continuous with respect to $(\tau, \beta)$ on $D(\varepsilon)$. 
Proof. Let $\left(\tau_{0}, \beta_{0}\right)$ is any point of $D(\varepsilon)$. In view of Theorems 2.2 and 3.1 we get

$$
\begin{aligned}
\lim _{\substack{\tau \rightarrow \tau_{0} \\
\beta \rightarrow \beta_{0}}} \varepsilon_{n}(\tau, \beta) & =\lim _{\substack{\tau \rightarrow \tau_{0} \\
\beta \rightarrow \beta_{0}}} \frac{\tau^{n-1}}{2 \pi} \int_{-\infty}^{\infty} \int_{-\infty}^{\infty}\left[\exp (-r) / r^{n+1}\right] \exp (-i \beta x) d x d y \\
& =\frac{\tau_{0}^{n-1}}{2 \pi} \int_{-\infty}^{\infty} \int_{-\infty}^{\infty} \lim _{\substack{\tau \rightarrow \tau_{0} \\
\beta \rightarrow \beta_{0}}}^{\infty}\left[\exp (-r) / r^{n+1}\right] \exp (-i \beta x) d x d y \\
& =\frac{\tau_{0}^{n-1}}{2 \pi} \int_{-\infty}^{\infty} \int_{-\infty}^{\infty} \frac{\exp \left(-\sqrt{x^{2}+y^{2}+\tau_{0}^{2}}\right)}{\left(\sqrt{x^{2}+y^{2}+\tau_{0}^{2}}\right)^{n+1}} \exp \left(-i \beta_{0} x\right) d x d y \\
& =\varepsilon_{n}\left(\tau_{0}, \beta_{0}\right) .
\end{aligned}
$$

Therefore the function $\varepsilon_{n}(\tau, \beta)$ is continuous on $D(\varepsilon)$.

3.4. Theorem. [18]. If $\xi$ is a scalar variable running through a closed interval $[a, b]$ and the function $f(\xi, \eta)$ is continuous together with its partial derivative $\frac{\partial f}{\partial \xi}$ on $[a, b] \times \bar{D}$ and if integral (3.1) is convergent while the integral

$$
F_{1}(\xi)=\int_{D} \frac{\partial f}{\partial \xi} f(\xi, \eta) d \eta
$$

is uniformly convergent with respect to $\xi \in[a, b]$ then $F^{\prime}(\xi)=F_{1}(\xi)$, that is

$$
\frac{\partial f}{\partial \xi} \int_{D} f(\xi, \eta) d \eta=\int_{D} \frac{\partial f}{\partial \xi} f(\xi, \eta) d \eta .
$$

3.5. Definition. [23]. Suppose $f: \mathbb{R} \longrightarrow \mathbb{C}$ is a locally integrable function on $\mathbb{R}$.

The function

$$
\hat{f}(\beta):=\frac{1}{\sqrt{2 \pi}} \int_{\mathbb{R}} f(x) \exp (-i \beta x) d x
$$

is called Fourier transform of the function $f$.

3.6. Theorem. [18]. If $f \in L=L(\mathbb{R})$, then $\hat{f}$, as bounded continuous functions, possess the property

$$
\lim _{|\beta| \rightarrow \infty} \hat{f}(\beta)=0
$$

3.7. Theorem. $\varepsilon_{n}(\tau, \beta)$ satisfies the following asymptotic equations:

$$
\begin{aligned}
& \varepsilon_{n}(\tau, \beta)=o(1), \quad(\tau, \beta) \in D(\varepsilon), \tau \rightarrow \infty . \\
& \varepsilon_{n}(\tau, \beta)=o(1), \quad(\tau, \beta) \in D(\varepsilon), \beta \rightarrow \pm \infty . \\
& \varepsilon_{n}(\tau, \beta)=E_{n}(\tau)+o(1), \quad(\tau, \beta) \in D(\varepsilon), \beta \rightarrow \infty .
\end{aligned}
$$


Proof. Using Theorem 2.2 and 3.1, we obtain that, for all $(\tau, \beta) \in D(\varepsilon)$

$$
\begin{aligned}
\lim _{\tau \rightarrow \infty} \varepsilon_{n}(\tau, \beta) & =\lim _{\tau \rightarrow \infty} \frac{\tau^{n-1}}{2 \pi} \int_{-\infty}^{\infty} \int_{-\infty}^{\infty}\left[\exp (-r) / r^{n+1}\right] \exp (-i \beta x) d x d y \\
& <\lim _{\tau \rightarrow \infty} \frac{1}{2 \pi} \int_{-\infty}^{\infty} \int_{-\infty}^{\infty}\left[\exp \left(-\sqrt{x^{2}+y^{2}+\tau^{2}}\right) / \tau^{2}\right] \exp (-i \beta x) d x d y \\
& =\frac{1}{2 \pi} \int_{-\infty}^{\infty} \int_{-\infty}^{\infty} \lim _{\tau \rightarrow \infty}\left[\exp \left(-\sqrt{x^{2}+y^{2}+\tau^{2}}\right) / \tau^{2}\right] \exp (-i \beta x) d x d y \\
& =0
\end{aligned}
$$

(3.2) holds.

Let us prove (3.3)

$$
\begin{aligned}
\varepsilon_{n}(\tau, \beta) & =\frac{\tau^{n-1}}{2 \pi} \int_{-\infty}^{\infty} \int_{-\infty}^{\infty}\left[\exp (-r) / r^{n+1}\right] \exp (-i \beta x) d x d y \\
& <\frac{\tau^{n-1}}{\sqrt{2 \pi}} \int_{-\infty}^{\infty}\left(\frac{1}{\sqrt{2 \pi}} \int_{-\infty}^{\infty}\left[\exp \left(-\sqrt{x^{2}+\tau^{2}}\right) /\left(\sqrt{x^{2}+\tau^{2}}\right)^{n+1}\right] \exp (-i \beta x) d x\right) d y \\
& =\frac{\tau^{n-1}}{\sqrt{2 \pi}} \int_{-\infty}^{\infty}\left(\frac{1}{\sqrt{2 \pi}} \int_{-\infty}^{\infty} f(\tau, x) \exp (-i \beta x) d x\right) d y
\end{aligned}
$$

where

$$
f(\tau, x)=\exp \left(-\sqrt{x^{2}+\tau^{2}}\right) /\left(\sqrt{x^{2}+\tau^{2}}\right)^{n+1} .
$$

Then from the Definition 3.1 we get

$$
\varepsilon_{n}(\tau, \beta)=\frac{\tau^{n-1}}{\sqrt{2 \pi}} \int_{-\infty}^{\infty} \hat{f}(\tau, \beta) d y
$$

and due to the following expression

$$
\begin{aligned}
& \int_{-\infty}^{\infty}|f(\tau, x)| d x=\int_{-\infty}^{\infty} \exp \left(-\sqrt{x^{2}+\tau^{2}}\right) /\left(\sqrt{x^{2}+\tau^{2}}\right)^{n+1} d x \\
< & 2 \exp (-\tau) \int_{\tau}^{\infty} 1 / u^{n} \sqrt{u^{2}-\tau^{2}} d u<\infty,
\end{aligned}
$$

$f \in L(\mathbb{R})$ by virtue of Theorem 3.5

$$
\lim _{|\beta| \rightarrow \infty} \hat{f}(\tau, \beta)=0 .
$$

From the Theorem 2.2, 3.1 and (3.5), (3.6) we get

$$
\begin{aligned}
\lim _{|\beta| \rightarrow \infty} \varepsilon_{n}(\tau, \beta) & =\frac{\tau^{n-1}}{\sqrt{2 \pi}} \int_{-\infty}^{\infty} \lim _{|\beta| \rightarrow \infty} \hat{f}(\tau, \beta) d y \\
& =0
\end{aligned}
$$

i.e., (3.3) holds. 
According to Theorem 2.2, 3.1 we obtain,

$$
\begin{aligned}
\lim _{\beta \rightarrow 0} \varepsilon_{n}(\tau, \beta) & =\lim _{\beta \rightarrow 0} \frac{\tau^{n-1}}{2 \pi} \int_{-\infty}^{\infty} \int_{-\infty}^{\infty}\left[\exp (-r) / r^{n+1}\right] \exp (-i \beta x) d x d y \\
& =\frac{\tau^{n-1}}{2 \pi} \int_{-\infty}^{\infty} \int_{-\infty}^{\infty}\left[\exp (-r) / r^{n+1}\right] \lim _{\beta \rightarrow 0} \exp (-i \beta x) d x d y \\
& =\frac{\tau^{n-1}}{2 \pi} \int_{-\infty}^{\infty} \int_{-\infty}^{\infty} \exp (-r) / r^{n+1} d x d y \\
& =\frac{\tau^{n-1}}{2 \pi} \int_{-\infty}^{\infty} \int_{-\infty}^{\infty} \int_{1}^{\infty} \exp (-t r) / r^{n} d t d x d y \\
& =\frac{\tau^{n-1}}{2 \pi} \int_{-\infty}^{\infty} \int_{-\infty}^{\infty} \phi(\tau, x, y) d x d y
\end{aligned}
$$

where

$$
\phi(\tau, x, y)=\int_{1}^{\infty} \exp (-t r) / r^{n} d t .
$$

It is clear that last integral is uniformly convergent with respect to $(\tau, x, y)$ on the domain $\left\{\tau \in[\varepsilon, \infty),(x, y) \in \mathbb{R}^{2}\right\}$.

So making use of the Theorem 3.2 we have

$$
\lim _{\beta \rightarrow 0} \varepsilon_{n}(\tau, \beta)=\frac{\tau^{n-1}}{2 \pi} \int_{1}^{\infty} \int_{-\infty}^{\infty} \int_{-\infty}^{\infty}(t-1)^{n-n} \exp (-t r) / r^{n} d x d y d t
$$

using the Theorem 2.2, 3.4 and (n-1) times integration by parts yield

$$
\begin{gathered}
\lim _{\beta \rightarrow 0} \varepsilon_{n}(\tau, \beta)=\frac{\tau^{n-1}}{(n-1) !} \frac{1}{2 \pi} \int_{1}^{\infty}(t-1)^{n-1} \int_{-\infty}^{\infty} \int_{-\infty}^{\infty} \exp (-t r) / r d x d y d t \\
=\frac{\tau^{n-1}}{(n-1) !} \int_{1}^{\infty}(t-1)^{n-1} \exp (-\tau t) / t d t .
\end{gathered}
$$

The last term can be written in the following form

$$
\begin{aligned}
\int_{1}^{\infty}(t-1)^{n-1} \exp (-\tau t) / t d t= & \int_{1}^{\infty}(t-1)^{n-2} \exp (-\tau t) d t-\int_{1}^{\infty}(t-1)^{n-3} \exp (-t \tau) d t \\
& +\int_{1}^{\infty}(t-1)^{n-4} \exp (-t \tau) d t \\
& -\ldots+(-1)^{n+1} \int_{1}^{\infty}(t-1) \exp (-t \tau) d t \\
& +(-1)^{n} \int_{1}^{\infty}(t-1) \exp (-t \tau) / t d t
\end{aligned}
$$


If we use integration by parts for all term we get

$$
\begin{aligned}
\int_{1}^{\infty}(t-1)^{n-1} \exp (-\tau t) / t d t= & {\left[\frac{(n-2) !}{\tau^{n-1}}-\frac{(n-3) !}{\tau^{n-2}}+\frac{(n-4) !}{\tau^{n-3}}\right.} \\
& \left.-\ldots+(-1)^{n} \frac{2 !}{\tau^{3}}+(-1)^{n-1} \frac{1 !}{\tau^{2}}+(-1)^{n} \frac{0 !}{\tau}\right] \exp (-\tau) \\
& +(-1)^{n-1} \int_{1}^{\infty} \exp (-\tau t) / t d t
\end{aligned}
$$

By means of integration by parts for the right-hand side of (3.8) then

$$
\begin{aligned}
(-1)^{n-1} \int_{1}^{\infty} \exp (-t \tau) / t d t= & (-1)^{n-1}\left[\frac{0 !}{\tau}-\frac{1 !}{\tau^{2}}+\frac{2 !}{\tau^{3}}-\ldots+(-1)^{n} \frac{(n-4) !}{\tau^{n-3}}\right. \\
& \left.+(-1)^{n-1} \frac{(n-3) !}{\tau^{n-2}}+(-1)^{n} \frac{(n-2) !}{\tau^{n-1}}\right] \exp (-\tau) \\
& +\frac{(n-1) !}{\tau^{n-1}} \int_{1}^{\infty} \exp (-t \tau) / t^{n} d t \text { label } 3.9
\end{aligned}
$$

substitution of (3.9) into (3.8) gives

$$
\int_{1}^{\infty}(t-1)^{n-1} \exp (-\tau t) / t d t=(n-1) ! / \tau^{n-1} \int_{1}^{\infty} \exp (-\tau t) / t^{n} d t
$$

considering the substitution of (3.10) into (3.7) gives

$$
\begin{aligned}
\lim _{\beta \rightarrow 0} \varepsilon_{n}(\tau, \beta) & =\int_{1}^{\infty} \exp (-\tau t) / t^{n} d t \\
& =E_{n}(\tau) .
\end{aligned}
$$

\section{Numerical Computation}

The numerical computation of TDEI functions have been studied by several authors. Those computation methods consist asymptotic or binomial series for TDEI function which include mass computatious [6]-[9], [11]- [16], [18]-[23]. On the basis of the uniform convergence of $\varepsilon_{n}(\tau, \beta)$, obtained in this paper we constructed a new simple and an accurate algorithm for the calculation of TDEI function even in a modarate PC. The computations were performed for large values of parameters $\tau$ and $\beta$. In this paper the TDEI functions were calculated on the Mathematica 8.0 international mathematical software. The comparative examples of computer calculatious for the TDEI functions are given in Tables 1-4. As can be seen from tables, our computational results are in agreement with literature [7]. Also from Tables 1-4 we see that the calculation results of the TDEI functions show good rate of convergence in the range of parameters $\tau \in$ $\left[10^{-3}, 1\right]$ and $\beta \in[1,20]$. 


\begin{tabular}{|c|c|c|c|c|c|}
\hline$\tau$ & $\beta=1$ & $\beta=2$ & $\beta=5$ & $\beta=10$ & $\beta=20$ \\
\hline 0.001 & $\begin{array}{c}A=6.143314628912719 \\
+i E-1 \\
B=6.1433\end{array}$ & $\begin{array}{c}A=5.850334088713723 \\
-i 9.375391894121577 E-10 \\
B=5.8503\end{array}$ & $\begin{array}{c}A=5.216596350515581 \\
-i 6.184411880623505 E-17 \\
B=5.2166\end{array}$ & $\begin{array}{c}A=4.622405662773734 \\
+i 4.417437057588218 E-18 \\
B=4.6224\end{array}$ & $\begin{array}{c}A=3.979468204969179 \\
+i 9.718361526694078 E-17 \\
B=3.9795\end{array}$ \\
\hline 0.005 & $\begin{array}{c}A=4.537901067889449 \\
+i 1.766974823035287 E-17 \\
B=4.5379\end{array}$ & $\begin{array}{c}A=4.245006981521351 \\
\quad+i E-1 \\
B=4.2450\end{array}$ & $\begin{array}{c}A=3.6118092517793587 \\
-i 1.018351544013959 E-10 \\
B=3.6118\end{array}$ & $\begin{array}{c}A=3.0192768074691063 \\
+i 9.055745968055847 E-17 \\
B=3.0193\end{array}$ & $\begin{array}{c}A=2.381847868201705 \\
+i 1.987846675914698 E-17 \\
B=2.3818\end{array}$ \\
\hline 0.01 & $\begin{array}{c}A=3.849813982491566 \\
i-3.837162415267503 E-11 \\
B=3.8498\end{array}$ & $\begin{array}{c}A=3.557142202395409 \\
-i 4.966478929026777 E-13 \\
B=3.5571\end{array}$ & $\begin{array}{c}A=2.9252964348968837 \\
-i 2.298302965135789 E-11 \\
B=2.9253\end{array}$ & $\begin{array}{c}A=2.3367504277586297 \\
-i 1.558030050211364 E-12 \\
B=2.3368\end{array}$ & $\begin{array}{c}A=1.7117877639753523 \\
-i 3.533949646070574 E-17 \\
B=1.7118\end{array}$ \\
\hline 0.025 & $\begin{array}{c}A=2.9488329356872187 \\
+i 1.553338671316446 E-10 \\
B=2.9488\end{array}$ & $\begin{array}{c}A=2.657383100971331 \\
+i 1.9939337580704 E-12 \\
B=2.6574\end{array}$ & $\begin{array}{c}A=2.0326700716719333 \\
-9.81770526868218 E-12 \\
B=2.0327\end{array}$ & $\begin{array}{c}A=1.4637881869087266 \\
-i 1.071335664531752 E-11 \\
B=1.4638\end{array}$ & $\begin{array}{c}A=8.941114707500399 E-1 \\
-i 5.300924469105861 E-17 \\
B=8.9411 E-1\end{array}$ \\
\hline 0.05 & $\begin{array}{c}A=2.281453068639485 \\
-i 5.300924469105861 E-17 \\
B=2.2815\end{array}$ & $\begin{array}{r}A=1.993344 \\
+i 1.046233699 \\
B=1\end{array}$ & $\begin{array}{r}A=1.38702 \\
-i 1.026298209 \\
B=1\end{array}$ & $\begin{array}{r}A=8.63916 \\
-i 4.310311 \\
B=8\end{array}$ & $\begin{array}{r}A=4.026457 \\
-i 2.69618961 \\
B=4.0\end{array}$ \\
\hline 0.1 & $\begin{array}{c}A=1.64017231138455 \\
\quad+i E-1 \\
B=1.6402\end{array}$ & $\begin{array}{c}A=1.3617758308830468 \\
+i 7.0578181485961 E-12 \\
B=1.3618\end{array}$ & $\begin{array}{c}A=8.043034952598128 E-1 \\
-i 1.985758332545723 E-12 \\
B=8.0430 E-1\end{array}$ & $\begin{array}{c}A=3.8435848086903923 E-1 \\
+i 8.28269448297791 E-17 \\
B=3.8436 E-1\end{array}$ & $\begin{array}{c}A=1.0713555332477835 E-1 \\
+i 6.092564529322131 E-12 \\
B=1.0714 E-1\end{array}$ \\
\hline 0.5 & $\begin{array}{c}A=4.2370709749072205 E-1 \\
+i E-1 \\
B=4.2371 E-1\end{array}$ & $\begin{array}{c}A=2.5067102641438316 E-1 \\
+i E-1 \\
B=2.5067 E-1\end{array}$ & $\begin{array}{c}A=4.630293776083369 E-2 \\
+i E-1 \\
B=4.6303 E-2\end{array}$ & $\begin{array}{c}A=3.0239699124587406 E-3 \\
+i 1.22467029569061 E-16 \\
B=3.0240 E-3\end{array}$ & $\begin{array}{c}A=1.552042299070299 E-5 \\
+i E-1 \\
B=1.5520 E-5\end{array}$ \\
\hline 1 & $\begin{array}{c}A=1.3554692860259693 E-1 \\
+i E-1 \\
B=1.3555 E-1\end{array}$ & $\begin{array}{c}A=5.351461855900747 E-2 \\
+i E-1 \\
B=5.3515 E-2\end{array}$ & $\begin{array}{c}A=2.3951848541335623 E-3 \\
+i E-1 \\
B=2.3952 E-3\end{array}$ & $\begin{array}{c}A=1.3321816513904781 E-5 \\
+i 7.07480153754363 E-18 \\
B=1.3322 E-5\end{array}$ & $\begin{array}{c}A=4.719616145834231 E-10 \\
-i 9.101430204813521 E-16 \\
B=4.7196 E-10\end{array}$ \\
\hline
\end{tabular}

Table 1. Value of $\varepsilon_{1}(\tau, \beta)$ 


\begin{tabular}{|c|c|c|c|c|c|}
\hline$\tau$ & $\beta=1$ & $\beta=2$ & $\beta=5$ & $\beta=10$ & $\beta=20$ \\
\hline 0.001 & $\begin{array}{c}A=9.924434723782973 E-1 \\
+i 1.413579858428229 E-19 \\
B=9.9244 E-1\end{array}$ & $\begin{array}{c}A=9.919160966891798 E-1 \\
+i E-1 \\
B=9.9192 E-1\end{array}$ & $\begin{array}{c}A=9.896973641980313 E-1 \\
-i 4.915307297290383 E-10 \\
B=9.8970 E-1\end{array}$ & $\begin{array}{c}A=9.853780518462402 E-1 \\
+i 1.413579858428229 E-19 \\
B=9.8538 E-1\end{array}$ & $\begin{array}{c}A=9.761947163307825 E-1 \\
+i 6.069070914791562 E-11 \\
B=9.7619 E-1\end{array}$ \\
\hline & $A=9.702643422545342 E-1$ & $A=9.676568670129494 E-1$ & $A=9.567681149630682 E-1$ & $A=9.35895855570027 E-1$ & $A=8.928151526927118 E-1$ \\
\hline 0.005 & $\begin{array}{c}+i 2.208718528794109 E-20 \\
B=9.7026 E-1\end{array}$ & $\begin{array}{c}+i 1.413579858428229 E-18 \\
B=9.6766 E-1\end{array}$ & $\begin{array}{c}+i 7.112741170587211 E-11 \\
B=9.5677 E-1\end{array}$ & $\begin{array}{c}-7.619368247065852 E-11 \\
B=9.3590 E-1\end{array}$ & $\begin{array}{c}-3.180554681463517 E-18 \\
B=8.9282 E-1\end{array}$ \\
\hline 0.01 & $\begin{array}{c}A=9.474592559549745 E-1 \\
-i 4.586072717261649 E-15 \\
B=9.4746 E-1\end{array}$ & $\begin{array}{c}A=9.423160462390637 E-1 \\
+i 3.533949646070575 E-18 \\
B=9.4232 E-1\end{array}$ & $\begin{array}{c}A=9.210350252606401 E-1 \\
+i 3.577803230650029 E-12 \\
B=9.2104 E-1\end{array}$ & $\begin{array}{c}A=8.810187337876387 E-1 \\
+i 2.827159716856459 E-18 \\
B=8.8102 E-1\end{array}$ & $\begin{array}{c}A=8.014083467390319 E-1 \\
-i 1.464519149540784 E-11 \\
B=8.0141 E-1\end{array}$ \\
\hline 0.025 & $\begin{array}{c}A=8.915415485850164 E-1 \\
+i 4.417437057588218 E-19 \\
B=8.9154 E-1\end{array}$ & $\begin{array}{c}A=8.791975192541199 E-1 \\
+i 3.639194635321662 E-13 \\
B=8.7920 E-1\end{array}$ & $\begin{array}{c}A=8.294982431226492 E-1 \\
+i 1.435944127455608 E-12 \\
B=8.2950 E-1\end{array}$ & $\begin{array}{c}A=7.412356049804204 E-1 \\
-i 2.8175139356756 E-13 \\
B=7.4124 E-1\end{array}$ & $\begin{array}{c}A=5.837991466302784 E-1 \\
+i 1.250068888192043 E-12 \\
B=5.8380 E-1\end{array}$ \\
\hline 0.05 & $\begin{array}{c}A=8.176587748648632 E-1 \\
+i 2.255574835843579 E-13 \\
B=8.1766 E-1\end{array}$ & $\begin{array}{c}A=7.945528161372075 E-1 \\
+i 1.766974823035287 E-18 \\
B=7.9455 E-1\end{array}$ & $\begin{array}{c}A=7.056034194084425 E-1 \\
-i 8.834874115176437 E-18 \\
B=7.0560 E-1\end{array}$ & $\begin{array}{c}A=5.618241448033833 E-1 \\
+i 4.594134539891746 E-17 \\
B=5.6182 E-1\end{array}$ & $\begin{array}{c}A=3.472878771358602 E-1 \\
-i 1.943672305338816 E-17 \\
B=3.4729 E-1\end{array}$ \\
\hline 0.1 & $\begin{array}{c}A=7.041062167438795 E-1 \\
-i 7.496678717427851 E-13 \\
B=7.0411 E-1\end{array}$ & $\begin{array}{r}A=6.634519 \\
-i 3.9097056 \\
B=6 .\end{array}$ & $\begin{array}{c}A=5.201241 \\
+i 1.8553235 \\
B=5\end{array}$ & $\begin{array}{r}A=3.2761 \\
+i 1.060184 \\
B=3\end{array}$ & $\begin{array}{c}A=1.2428402294804283 E-1 \\
+i E-1 \\
B=1.2428 E-1\end{array}$ \\
\hline 0.5 & $\begin{array}{c}A=2.812151426498713 E-1 \\
+i E-1 \\
B=2.8122 E-1\end{array}$ & $\begin{array}{c}A=2.015863821445704 E-1 \\
+i E-1 \\
B=2.0159 E-1\end{array}$ & $\begin{array}{c}A=5.496848551136895 E-2 \\
\quad+i E-1 \\
B=5.4968 E-2\end{array}$ & $\begin{array}{c}A=5.0600102554520555 E-3 \\
-i 3.224667578916283 E-15 \\
B=5.0600 E-3\end{array}$ & $\begin{array}{c}A=3.7076101127100064 E-5 \\
+i E-1 \\
B=3.7076 E-5\end{array}$ \\
\hline 1 & $\begin{array}{c}A=1.0756980583160117 E-1 \\
+i E-1 \\
B=1.0757 E-1\end{array}$ & $\begin{array}{c}A=5.336330710138579 E-2 \\
\quad+i E-1 \\
B=5.3363 E-2\end{array}$ & $\begin{array}{c}A=3.7075424200455013 E-3 \\
+i E-1 \\
B=3.7075 E-3\end{array}$ & $\begin{array}{c}A=2.9869304279352523 E-5 \\
-i 5.866908592109352 E-19 \\
B=2.9869 E-5\end{array}$ & $\begin{array}{c}A=1.538334495203213 E-9 \\
-i 2.63033517911171 E-18 \\
B=1.5383 E-9\end{array}$ \\
\hline
\end{tabular}

Table 2. Value of $\varepsilon_{2}(\tau, \beta)$ 


\begin{tabular}{|c|c|c|c|c|c|}
\hline$\tau$ & $\beta=1$ & $\beta=2$ & $\beta=5$ & $\beta=10$ & $\beta=20$ \\
\hline 0.001 & $\begin{array}{c}A=4.990024511109741 E-1 \\
-i 2.261727773485167 E-21 \\
B=4.9900 E-1\end{array}$ & $\begin{array}{c}A=4.989982320156957 E-1 \\
-i 1.776096495894175 E-12 \\
B=4.9900 E-1\end{array}$ & $\begin{array}{c}A=4.989706876695545 E-1 \\
-i 2.714073328182201 E-19 \\
B=4.9897 E-1\end{array}$ & $\begin{array}{c}A=4.9888175736258644 E-1 \\
-i 1.442151075825898 E-9 \\
B=4.9888 E-1\end{array}$ & $\begin{array}{c}A=4.9856897323705635 E-1 \\
+i 5.790023100122029 E-19 \\
B=4.9857 E-1\end{array}$ \\
\hline 0.005 & $\begin{array}{c}A=4.950516902549029 E-1 \\
-i 2.544443745170813 E-19 \\
B=4.9505 E-1\end{array}$ & $\begin{array}{c}A=4.949762046326981 E-1 \\
-i 1.90159059838535 E-13 \\
B=4.9498 E-1\end{array}$ & $\begin{array}{c}A=4.9449751991327523 E-1 \\
+i 2.261727773485167 E-19 \\
B=4.9450 E-1\end{array}$ & $\begin{array}{c}A=4.930242429216283 E-1 \\
-i 1.133479574912619 E-13 \\
B=4.9302 E-1\end{array}$ & $\begin{array}{c}A=4.8819820352499177 E-1 \\
-i 7.237528875152536 E-18 \\
B=4.8820 E-1\end{array}$ \\
\hline 0.01 & $\begin{array}{c}A=4.9018922746454213 E-1 \\
+i 4.229134084646993 E-15 \\
B=4.9019 E-1\end{array}$ & $\begin{array}{c}A=4.899385430768269 E-1 \\
+i 8.469661622952918 E-15 \\
B=4.8994 E-1\end{array}$ & $\begin{array}{c}A=4.883821102053627 E-1 \\
+i 1.809382218788134 E-18 \\
B=4.8838 E-1\end{array}$ & $\begin{array}{c}A=4.837637107484798 E-1 \\
+i E-1 \\
B=4.8376 E-1\end{array}$ & $\begin{array}{c}A=4.69496901043039 E-1 \\
-i 1.970457721187423 E-12 \\
B=4.6950 E-1\end{array}$ \\
\hline 0.025 & $\begin{array}{c}A=4.760365743919093 E-1 \\
+i 8.834874115176437 E-20 \\
B=4.7604 E-1\end{array}$ & $\begin{array}{c}A=4.748853995162321 E-1 \\
-i 6.626155586382327 E-19 \\
B=4.7489 E-1\end{array}$ & $\begin{array}{c}A=4.6805408708589463 E-1 \\
+i 2.827159716856459 E-18 \\
B=4.6805 E-1\end{array}$ & $\begin{array}{c}A=4.4938184179542284 E-1 \\
-i 3.180554681463517 E-18 \\
B=4.4938 E-1\end{array}$ & $\begin{array}{c}A=3.9923273619928473 E-1 \\
+2.216955693995738 E-12 \\
B=3.9923 E-1\end{array}$ \\
\hline 0.05 & $\begin{array}{c}A=4.536942695174573 E-1 \\
+i 4.28843815505793 E-12 \\
B=4.5369 E-1\end{array}$ & $\begin{array}{c}A=4.5029816049684535 E-1 \\
-i 8.607344654558792 E-12 \\
B=4.5030 E-1\end{array}$ & $\begin{array}{c}A=4.313003524176357 E-1 \\
+i 6.523616104772877 E-13 \\
B=4.3130 E-1\end{array}$ & $\begin{array}{c}A=3.849140185306733 E-1 \\
+i 1.711286844512507 E-13 \\
B=3.8491 E-1\end{array}$ & $\begin{array}{c}A=2.8307826133448905 E-1 \\
+i 1.753696007240177 E-12 \\
B=2.8308 E-1\end{array}$ \\
\hline 0.1 & $\begin{array}{c}A=4.129052504262309 E-1 \\
+i 2.260774994155964 E-11 \\
B=4.1291 E-1\end{array}$ & $\begin{array}{c}A=4.0381514638243077 E-1 \\
-i 1.210519571178468 E-11 \\
B=4.0382 E-1\end{array}$ & $\begin{array}{c}A=3.5787773372208315 E-1 \\
+i E-1 \\
B=3.5788 E-1\end{array}$ & $\begin{array}{c}A=2.662095412797268 E-1 \\
+i E-1 \\
B=2.6621 E-1\end{array}$ & $\begin{array}{c}A=1.2689054916664513 E-1 \\
+i E-1 \\
B=1.2689 E-1\end{array}$ \\
\hline 0.5 & $\begin{array}{c}A=2.0269794527722496 E-1 \\
+i E-1 \\
B=2.0270 E-1\end{array}$ & $\begin{array}{c}A=1.6215841061428077 E-1 \\
+i E-1 \\
B=1.6216 E-1\end{array}$ & $\begin{array}{c}A=5.843514756064822 E-2 \\
+i E-1 \\
B=5.8435 E-2\end{array}$ & $\begin{array}{c}A=7.175956423511752 E-3 \\
-i 5.978291420340211 E-15 \\
B=7.1760 E-3\end{array}$ & $\begin{array}{c}A=7.267195676212158 E-5 \\
\quad+i E-1 \\
B=7.2672 E-5\end{array}$ \\
\hline 1 & $\begin{array}{c}A=8.726359085360859 E-2 \\
+i E-1 \\
B=8.7264 E-2\end{array}$ & $\begin{array}{c}A=5.059279476996788 E-2 \\
+i E-1 \\
B=5.0593 E-2\end{array}$ & $\begin{array}{c}A=4.997178810918784 E-3 \\
+i 1.104359264397054 E-18 \\
B=4.9972 E-3\end{array}$ & $\begin{array}{c}A=5.5981429368903135 E-5 \\
-i 3.337882737606331 E-19 \\
B=5.5981 E-5\end{array}$ & $\begin{array}{c}A=4.09183571344698 E-9 \\
+i E-1 \\
B=4.0918 E-9\end{array}$ \\
\hline
\end{tabular}

Table 3. Value of $\varepsilon_{3}(\tau, \beta)$ 


\begin{tabular}{|c|c|c|c|c|c|}
\hline$\tau$ & $\beta=1$ & $\beta=2$ & $\beta=5$ & $\beta=10$ & $\beta=20$ \\
\hline 0.01 & & $\begin{array}{l}36 E-1 \\
58 E-14\end{array}$ & $\begin{array}{c}3.279999313606849 E-1 \\
+i 7.237528875152537 E-19 \\
\end{array}$ & $\begin{array}{l}46 E-1 \\
48 E-11\end{array}$ & $\begin{array}{l}31525 E-1 \\
17758 E-17\end{array}$ \\
\hline 0.05 & & & $\begin{array}{r}3.020236108 \\
+i 2.261727773\end{array}$ & $\begin{array}{r}2.83299035 \\
+i 6.4323323\end{array}$ & $\begin{array}{c}2.3065645383776914 E-1 \\
-i 9.830608248932432 E-13\end{array}$ \\
\hline 0.1 & $\begin{array}{c}2.866606684383088 E-1 \\
+i 3.486063214786459 E-13\end{array}$ & $\begin{array}{c}2.835510053837311 E-1 \\
+i 7.067899292141149 E-20\end{array}$ & $\begin{array}{c}2.6473792976651495 E-1 \\
-i 1.953758586363162 E-12\end{array}$ & $\begin{array}{c}2.1692835774847108 E-1 \\
-i 5.654319433712919 E-18\end{array}$ & $\begin{array}{c}1.2200667108444715 E-1 \\
+i E-1\end{array}$ \\
\hline & $\begin{array}{c}7.2525046372837961 \\
+i E-1\end{array}$ & $\begin{array}{r}75137732958 \\
+i E-1 \\
\end{array}$ & $\begin{array}{c}6.173857960619937 E-3 \\
-i 5.521796321985272 E-19 \\
\end{array}$ & $\begin{array}{c}9.31476402535588 E-5 \\
+i 9.518492990879253 E-18 \\
\end{array}$ & $\begin{array}{c}9.484194215269618 E-9 \\
-i 2.229293299158516 E-21 \\
\end{array}$ \\
\hline
\end{tabular}

Table 4. Value of $\varepsilon_{4}(\tau, \beta)$ 


\section{References}

[1] Z. Altac, Integrals involving Bickley and Bessel functions in radiative transfer amd generalized exponential integral functions, ASME Journal of Heat Transfer, Volume 118, 1996, Pages 789-792.

[2] Z. Altac, Exact series expansions, recurrence relations, properties and integrals of the generalized exponential integral functions, Journal of Quantitative Spectroscopy and Radiative Transfer, Volume 104, Issue 3, April 2007, Pages 310-325.

[3] Y. Aygar, E. Bairamov, Properties of the two-dimensional exponential integral functions, Journal of Mathematical Chemistry, May 2011, Volume 49, Issue 5, pp 1014-1025.

[4] E. Bairamov, N. Ozalp, On convergence, asymptotic behaviour and computational algorithm of generalized exponential integral functions arising in a radiative transfer, Journal of Quantitative Spectroscopy and Radiative Transfer, Volume 113, Issue 14, September 2012, Pages 1818-1825.

[5] E. Bairamov, S. Yardimci, Uniform convergence and the properties of the exponential and generalized exponential integral functions, Journal of Quantitative Spectroscopy and Radiative Transfer, Volume 111, Issue 16, November 2010, Pages 2471-2473.

[6] W.F. Breig, A.L. Crosbie, Two-dimensional radiative equilibrium semi-infinite medium subjected to cosine varying radiation. J. Quantitative Spectroscopy \& Radiative Transfer 13 (1973) 1395-1419.

[7] W.F. Breig, A.L. Crosbie, Numerical computation of a generalized exponential integral function. Math. Comput. 28 (1974) 575-579.

[8] I.M. Busbridge, The mathematics of radiative transfer. Cambridge 1960.

[9] S. Chandrasekhar, Radiative Transfer, Dover Publications, New York, 1960.

[10] C. Chiccoli, S. Lorenzutta, G. Maino; Concerning some integrals of the generalized exponential-integral function. Computers \& Mathematics with Applications, Volume 23, Issue 11, June 1992, Pages 13-21.

[11] A.L. Crosbie, R.L. Dougherty, Two-dimensional in a cylindrical geometry with anisotropic scattering. J. Quantitative Spectroscopy \& Radiative Transfer, 25 (1981) 551-569.

[12] A.L. Crosbie, L.C. Lee, Relation between multidimensional radiative transfer in cylindrical and rectangular coordinates with anisotropic scattering. J. Quantitative Spectroscopy \& Radiative Transfer, 38 (1987) 231-241.

[13] A.L. Crosbie, L.C. Lee, Multidimensional radiative transfer: a single integral represantation of anisotropic scattering kernels J. Quantitative Spectroscopy \& Radiative Transfer, 42 (1989) 239-246.

[14] I.S. Gradshteyn, I.M. Ryzhik, Table of integrals, series and products Academic Press, NewYork, 2007.

[15] I.I. Guseinov, B.A. Mamedov, On the evalution of generalized exponential integral functions, J. Quantitative Spectroscopy \& Radiative Transfer, 102 (2006) 251-256.

[16] V. Kourganoff, Basic Methods in Transfer Problems, Dover Publications, New York, 1963.

[17] B.A. Mamedov; Evaluation of generalized exponential integral function using binomial expansion theorems, Journal of Quantitative Spectroscopy and Radiative Transfer, Volume 94, Issues 3-4, 1 September 2005, Pages 507-514.

[18] S.M. Nikolsky,. Course of Mathematical Analysis. Vol. 2, Mir Publishers, Moscow 1977.

[19] R.H. Prabha, R.D.S. Yadav, Polynomial expressions for Bickley and exponential integral functions. Ann. Nucl. Energy, 23 (1996) 1021-1025.

[20] V.V. Sobelev, A Treatise on Radiative Transfer Princeton, New York, 1963

[21] H.C. Van de Hulst, Multiple Light Scattering, Vol. 1, Academic Press, New York, 1980.

[22] W.W Yuen, C.F. Ho, Analysis of two-dimensional radiative heat transfer in a gray medium with internal heat generation, Int. J. Heat Mass Transfer, 28 (1985) 17-23.

[23] V.A. Zorich, Mathematical Analysis. Vol. 2, Springer, New York 2004. 
